\title{
Imaging pattern and histological features of Gorham-Stout Disease of the radius
}

\author{
Clémence Bruyère $^{* 1}$, Valentina Garibotto ${ }^{2}$, Anne-Laure Rougemont ${ }^{3}$, Sana Boudabbous ${ }^{1}$ \\ ${ }^{1}$ Radiology department, HUG, Switzerland \\ ${ }^{2}$ Nuclear Medecin department, HUG, Switzerland \\ ${ }^{3}$ Pathology department, HUG, Switzerland
}

Received: April 23, 2018

DOI: $10.5430 /$ jst.v8n2p20
Accepted: June 6, 2018

Online Published: June 20, 2018

\begin{abstract}
Gorham-Stout Disease (GD) is a very rare disease of unknown etiology characterized by progressive osteolysis and soft tissue involvement. Imaging is non-specific, and diagnosis may be delayed. The evolution of the disease is unpredictable, with progression of the osteolysis, spontaneous regression, or in a few cases re-ossification. We report a case of a 54-year-old woman with GD of the radius. In this rare location, only few cases have been reported with all imaging modalities: conventional radiography, ultrasound, computed tomography (CT), magnetic resonance imaging (MRI), and positron emission tomography (PET-CT). We describe the characteristics of GD in different imaging modalities, as well as the histological features. To the best of our knowledge, we report the first metabolically active lesion in GD, with relevant implications for the differential diagnosis.
\end{abstract}

Key Words: Gorham's Disease, Radius, Osteolysis, PET-CT

\section{INTRODUCTION}

Gorham-Stout Disease (GD) is a very rare disease characterized by local proliferation of small vascular or lymphatic channels resulting in progressive osteolysis and bone resorption. ${ }^{[1]}$ The evolution of the disease is unpredictable, some lesions showing progression of the osteolysis, while others undergo spontaneous regression. This lesion can be locally very aggressive. This entity is non-hereditary, has no gender or ethnic predilection, and can occur at any age from 1 month to 75 years, with a peak incidence before 40 years of age. ${ }^{[2-4]}$ Any part of the skeletal system may be affected, involving both axial and appendicular skeleton, but the shoulder, pelvis and mandible are the most common sites of involvement. ${ }^{[4,5]}$ Imaging features are non-specific and osteolytic feature is the common pattern in conventional and CT imaging. In advanced modalities (MRI with functional sequences) GD can mimic aggressive tumors. Only two previous cases evaluated by 18 F-Fluorodeoxyglucose (FDG) PET-CT have been reported, showing low metabolic activity in these lesions. ${ }^{[6,7]}$ No treatment modality has proven to give satisfactory results. The treatment of this disease includes three major categories: medicine therapy, radiation and surgery, but there is currently no single recommendation in the contemporary English literature. ${ }^{[2-5]}$

\section{CASE REPORT}

A 54-year-old woman with a medical history of acute myeloid leukemia (AML) in complete remission, presented with right upper limb pain, which was considered as a tendonitis. Persistence of the symptoms despite symptomatic

\footnotetext{
*Correspondence: Clémence Bruyère; Email: bruyereclemence@gmail.com; Address: Radiology department, HUG, 4 rue grabriel perret gentil 1204 Geneve, Switzerland.
} 
treatment was indication to an X-ray, performed 6 months after initial symptoms. Imaging showed an important osteolysis of the proximal metaphyso-diaphysis of the right radius (see Figure 1).

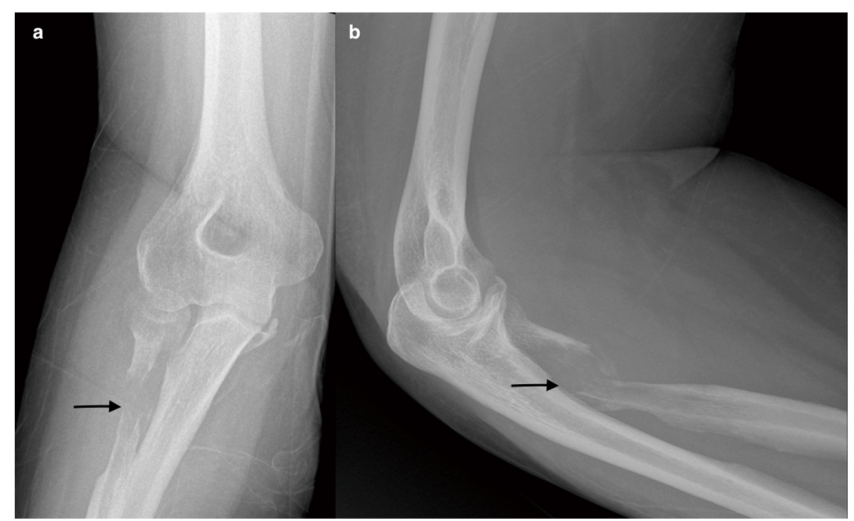

Figure 1. Antero-posterieur (a) and lateral (b) view of the elbow showed important osteolysis of the proximal metaphyso-diaphysis of the right radius (arrow)
Given the patient's medical history, a FDG PET-CT was performed one month later, to exclude leukemia recurrence or metastatic disease. The radial lesion showed hypermetabolic activity (maximum standardized uptake value -SUVmaxof 5,2) without any other pathological finding (see Figure 2). Additionally, a bone marrow biopsy was performed, and showed no malignancy. As all investigations remained negative, two consecutive percutaneous biopsies were performed, but showed only fibro-inflammatory modifications of the adipose tissue and skeletal muscle, and the absence of tumor. Patient MRI follow-up, one month thereafter, demonstrated a soft-tissue mass in hyposignal T1, hypersignal T2 and STIR surrounding a large area of osteolysis of the proximal right radius, without restriction of diffusion. The apparent diffusion coefficient (ADC) value was $1.9 \times 10^{-3} \mathrm{~mm}^{2} / \mathrm{sec}$ (see Figure 3). X-ray controls showed osteolysis progression (see Figure 4). The patient presented concurrent paralysis of the right extensor digitorum communis. Finally, through a multidisciplinary approach, decision was made to perform a surgical biopsy. Histology showed a proliferation of irregular, anastomosed or branched vessels. Endothelial cells showed no atypia, and there were no malignant cells in the interstitium.

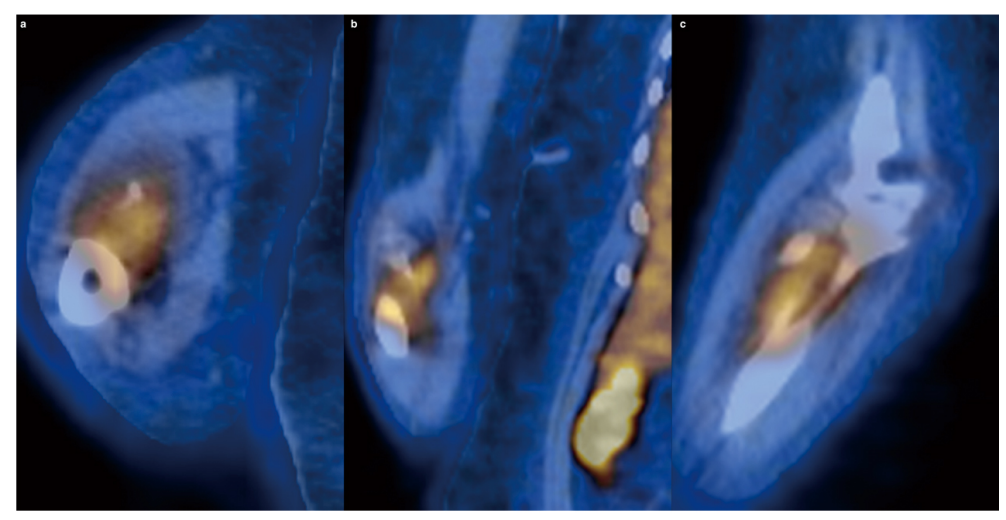

Figure 2. FDG PET-CT (axial (a), coronal (b), and sagittal (c) view) showed a hypermetabolic activity of the radial lesion (maximum standardized uptake value -SUVmax- of 5,2)

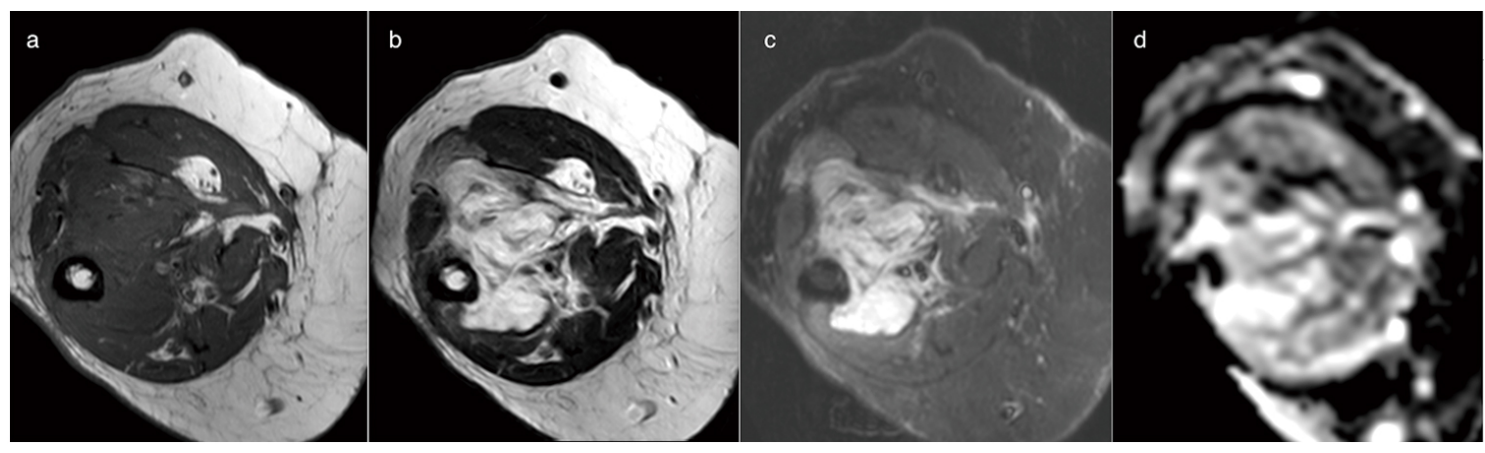

Figure 3. MRI demonstrated a soft-tissue mass in hyposignal T1 (a), hypersignal T2 (b) and STIR (c) surrounding a large area of osteolysis of the proximal right radius, without restriction of diffusion $\left(\mathrm{ADC}=1.9 \times 10^{-3} \mathrm{~mm}^{2} / \mathrm{sec}\right)(\mathrm{d})$ 
Fibrosis and chronic inflammation were seen, together with large areas of skeletal muscle atrophy. No bone was seen, in a surgical biopsy performed in the center of the "vanishing" radius. Histological findings are illustrated in Figure 5. Finally 11 months after initial presentation, GD was suspected based on the combination of the clinical, histological and radiological findings. Initial treatment combined calcitonin, vitamin D, and fractional radiotherapy. The patient was however thereafter lost to follow-up.

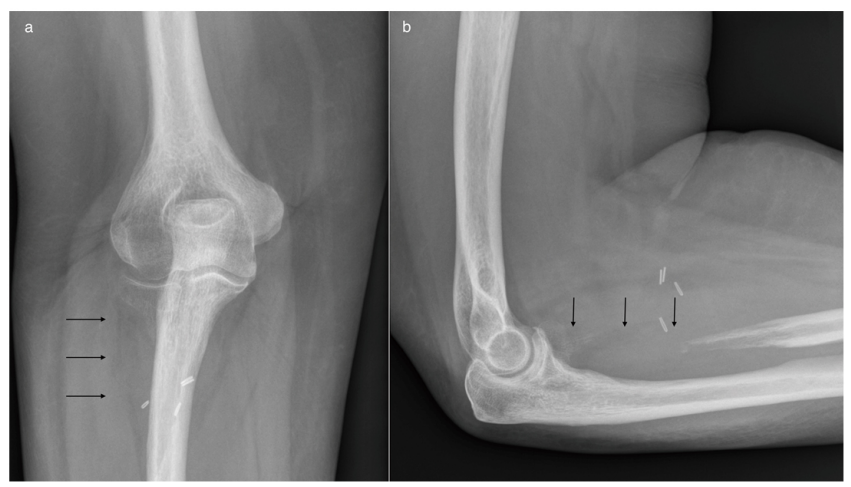

Figure 4. Antero-posterieur (a) and lateral (b) X-ray controls showed osteolysis progression (arrows) of the proximal epiphyso-metaphyso-diaphysis of the right radius

\section{Discussion}

This case illustrates the diagnostic difficulties in GD, and the consecutive delay in appropriate treatment initiation, despite the large investigative methods applied including biopsies. The pathogenesis and etiology of GD remain largely unknown. Only 200 cases have been reported in the literature until now. The first report of spontaneous bone resorption was described in 1838 and again in 1872 by Jackson. ${ }^{[8]}$ Gorham and Stout established the clinical features and pathological description in 1955. ${ }^{[9]}$ The diagnosis of GD is based on exclusion of differential diagnostic categories, and relies on a combination of the clinical, radiological and histopathological findings. ${ }^{[10]} \mathrm{Heffez}$ and all ${ }^{[11]}$ identified a number of criteria to help distinguish GD from other osteolytic processes (see Table 1): 1) A positive biopsy for angiomatous tissue, 2) Absence of cellular atypia, 3) Minimal or no osteoblastic response and absence of dystrophic calcification, 4) Evidence of locally progressive osseous resorption, 5) No expansile, no ulcerative lesion, 6) Absence of visceral involvement, 7) Osteolytic radiographic pattern, 8) Negative hereditary, metabolic, neoplastic, immunological or infectious etiology, 9) Spread to adjacent soft tissues, 10) Development of fibrous tissue. To the best of our knowledge, it's the first case of GD with AML. We didn't find any corre- lation between these two diseases in the literature. Usually, several modalities are used to establish the diagnosis. Since they are non-specific, we will describe only the most frequent findings. Radiographic appearance is the most dramatic aspect of the disease. ${ }^{[4]}$ In the initial stage, radiolucent foci appear in the intramedullary or subcortical regions. After that, slowly progressive atrophy, dissolution, fracture, fragmentation and disappearance of a portion of a bone occur, and in the same time, soft tissue atrophy appears. In some cases, the disease progresses to the adjacent bones; the joints provide no protection against the extension of the disease. ${ }^{[4]}$ No description of US findings was found in the literature. In our case a non-specific pseudomass was observed near the radius bone without hypervascularity. US may help to perform guided biopsy as in our case. Computed tomography shows the extension of osteolysis or bone defects and threedimensional CT reconstructions may be helpful in planning surgical excision and reconstruction. ${ }^{[10]}$ The MRI findings are non-specific and depend on the degree of neovascular progression and fibrosis. ${ }^{[5-12]}$ The signal on T1 and T2 weighted imaging is variable, becoming lower as the degree of fibrosis increases. The pattern of enhancement is also variable, a finding that could be attributed to the variable composition of hemangiomatous and/or lymphangiomatous tissus. ${ }^{[12]}$ To the best of our knowledge, diffusion sequence (DWI) in this disease is not described in the English literature, but is helpful for the diagnosis. No diffusion restriction is expected in this disease as no tumor proliferation is found. This sequence is important to exclude malignant tumor. MRI is still the preferred imaging technique to evaluate the extension of the disease regarding the sensitivity to detect the soft tissue involvement. ${ }^{[10]}$ Spieth and all, ${ }^{[13]}$ using three phase bone scintigraphy with $99 \mathrm{mTc}$-labelled methylene disphophonate and thallium imaging, demonstrated slightly decreased activity in the arterial phase, slightly increased activity in the blood pool phase, and increased activity in the delayed phase with normal thallium activity. Thallium imaging was performed to exclude malignant or soft tissue tumours. FDG PET/CT findings: only two previous cases have been reported $^{[6,7]}$ to the best of our knowledge: in both cases the lesion did not show any significant metabolic activity without mention of the SUV value. We remind that the critical threshold of SUV max depends on the basic metabolism of each patient to define a hypermetabolic activity for each disease. Our data suggest that metabolic activity in the lesion may be heterogeneous, and may possibly vary with disease phase. As previously mentioned, the progression of GD is unpredictable. Jing Shi et al. ${ }^{[5]}$ showed re-ossification of the wrist after treatment, with bone formation occurring in the lytic areas. 


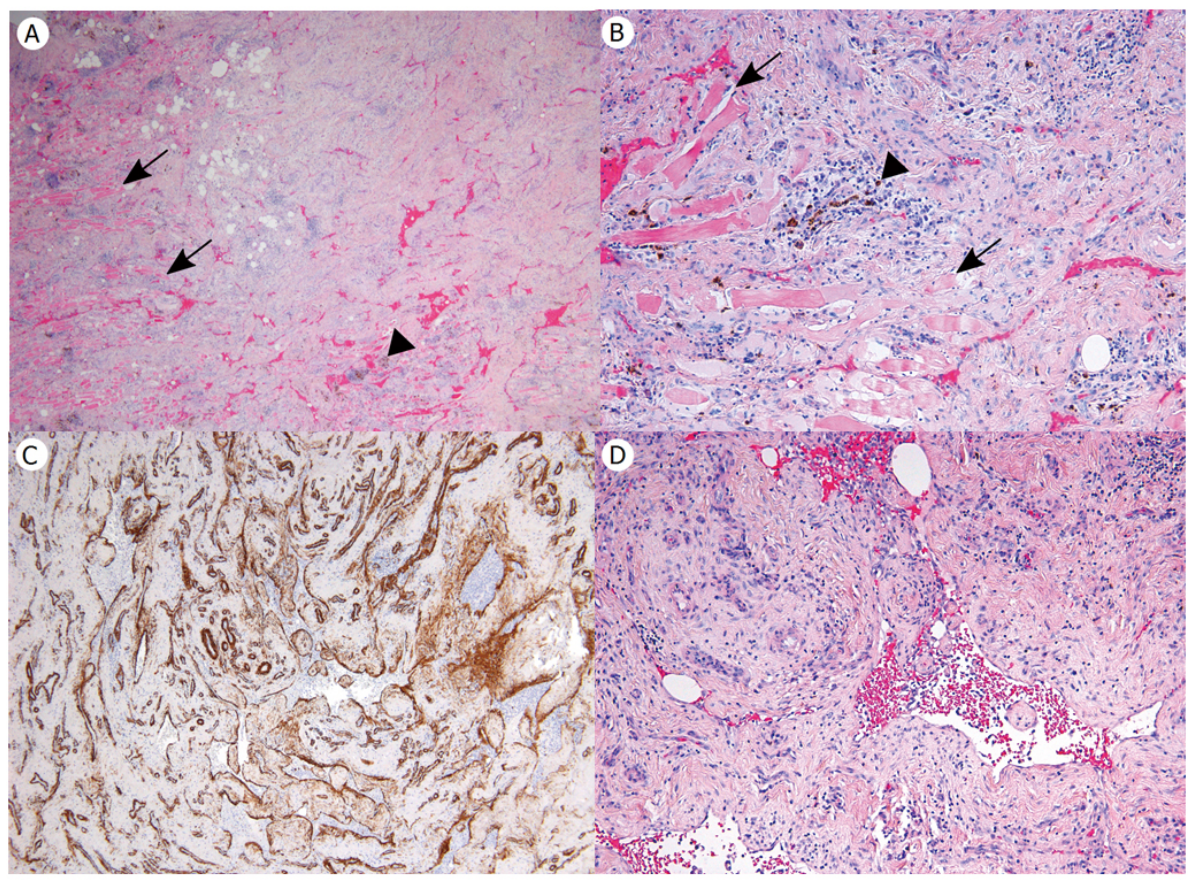

Figure 5. Histological findings, surgical biopsy from the center of the "vanishing" radius. A. Low magnification identifies a destructive fibro-inflammatory process extending to the adipose tissue and skeletal muscle. No residual bone is seen (Hematoxylin\&Eosin, H\&E, original magnification 20×). B. Higher magnification highlights the peripheral skeletal muscle atrophy, and accompanying fibrosis and chronic inflammatory component. Hemosiderin-laden macrophages are indicative of previous hemorrhage $(\mathrm{H} \& \mathrm{E}, 1000 \times)$. C. Smooth muscle actin helps identify the numerous vessels, highlighting the irregular muscular wall of the proliferating anastomosed or branched vessels (SMA, 400×). D. The vessels appear more in keeping with a malformation process, and endothelial cells show no atypia (H\&E, 1000×).

Table 1. Criteria to help distinguish Gorham disease from other osteolytic processes adapted from Heffez $\mathrm{L}$ and all ${ }^{[11]}$

1. A positive biopsy for angiomatous tissue
2. Absence of cellular atypia.
3. Minimal or no osteoblastic response and absence of dystrophic
calcification
4. Evidence of local progressive osseous resorption
5. No expansile, no ulcerative lesion
6. Absence of visceral involvement
7. Osteolytic radiographic pattern
8. Negative hereditary, metabolic, neoplastic, immunological or
infectious etiology
9. Spread to adjacent soft tissues
10. Development of fibrous tissue.

The treatment of GD is controversial. Several therapeutic modalities including surgery; radiation therapy, anti-

\section{REFERENCES}

[1] Liu Y, et al. Gorham-Stout disease: radiological, histological, and clinical features of 12 cases and review of literature. Clin Rheumatol. 2016; 35: 813-823. PMid:25227772. https://doi.org/10.100 7/s10067-014-2780-2

[2] Nikolaou V, Chytas D, Korres D, et al. Vanishing bone disease osteoclastic medications (bisphosphonates) and alpha-2b interferon have been used in the management of GD. But there is no consensus about the most efficient treatment of GD. ${ }^{[5]}$ $\mathrm{R}$. Heyd et al. ${ }^{[14]}$ showed that conventionally fractionated external beam radiotherapy with a total dose of 36 to $45 \mathrm{~Gy}$ might effectively prevent local disease progression in $77 \%$ to $80 \%$ of the cases. The proper course of treatment for GD remains uncertain. In summary, GD is a rare entity of as yet unknown etiology. Imaging, including metabolic imaging by FDG PET/CT, is non-specific and treatment guidelines remain to be defined. This rare and locally destructive diagnosis should however be kept in mind, in order to avoid treatment delay and control osteolysis progression.

\section{CONFlicts of InTEREST Disclosure}

The authors declare that they have no competing interests.
(Gorham-Stout syndrome): A review of a rare entity. World J Orthop. 2014 November 18; 5(5): 694-698.

[3] Boyer P, Bourgeois P, Saillant G, et al. Massive GorhamStout syndrome of the pelvis. Clin Rheumatol. 2005; 24: 551555. PMid:15827684. https://doi.org/10.1007/s10067-005 $-1088-7$ 
[4] Patel DV, et al. Gorham's Disease or Massive Osteolysis. Clinical Medicine \& Research. 2005; 3(2): 65-74. https://doi .org/10.3 $121 / \mathrm{cmr} .3 .2 .65$

[5] Shi J, Zhang Z, Gao F, et al. Reossification in Gorham's disease of the hand and wrist with unusual CT and MR imaging features. Skeletal Radiol. 2015; 44: 1033-1038. PMid:25712769. https : //doi.org/10.1007/s00256-015-2121-9

[6] Papadakis GZ, et al. 18F-NaF and 18F-FDG PET/CT in GorhamStout disease. Clin Nucl Med. 2016 November; 41(11): 884-885.

[7] Rohit M, et al. Vanishing bone disease involving the pelvis. J Cancer Res Ther. December 2005; 1(4).

[8] Jackson JBS. A boneless arm. Boston Med Surg J. 1838; 18: $368 \mathrm{e} 9$.

[9] Gorham LW, Stout AP. Massive osteolysis acute spontaneous absorption of bone, phantom bone, disappearing bone: its relation to haemangiomatosis. J Bone Joint Surg Am. 1955; 37: 985e1004.
[10] Kai B, Ryan A, Dunlop P, et al. Gorham disease of bone: three cases and review of radiological features. Skeletal Radiol. 2002; 31: 301-306.

[11] Heffez L, Doku HC, Carter BL, et al. Perspectives on massive osteolysis report of a case and review of the literature. Oral Surf Oral Med Oral Pathol. 1983; 55: 331-43. https ://doi.org/10.1016/ 0030-4220 (83) 90185-8

[12] Yoo SY, Hwan Hong S, Kang S, et al. MRI of Gorham's disease: findings in two cases. Skeletal Radiol. 2002; 31: 301306. PMid:11981608. https://doi.org/10.1007/s00256-002 -0487-y

[13] Spieth ME, et al. Gohram's disease of the radius: radiographic, scintigraphic and MRI findings with pathologic correlation. Skeletal Radiol. 1997.

[14] Heyd R, et al. Radiation therapy for Gohram stout syndrome: results of a national patterns of care study and literature review. I. J. Radiation Oncology. 2011; 8(3). 